\title{
Mecamylamine pretreatment increases subsequent nicotine self-administration as indicated by changes in plasma nicotine level
}

\author{
C.S. Pomerleau, O.F. Pomerleau, and M.J. Majchrzak \\ Behavioral Medicine Program, Department of Psychiatry, University of Michigan, Ann Arbor, MI 48105, USA
}

\begin{abstract}
Acute administration of mecamylamine, a centrally active nicotinic cholinergic agonist, has been shown to increase amount of smoking as indicated by smoking topography (e.g., puff rate, puff duration), expired carbon monoxide changes, and other inferential measures. In the present study, subjects showed significantly greater increases in plasma nicotine following smoking of two highnicotine research cigarettes when pretreated with mecamylamine than when pretreated with placebo, even though no significant differences in puff volume or puff number were detected. Interestingly, none of our subjects reported nausea, although some achieved plasma nicotine levels at which nausea would typically be expected. We attribute the observed increases in nicotine intake to compensatory behavior designed to overcome mecamylamine's blocking effects.
\end{abstract}

Key words: Cigarette smoking - Mecamylamine - Plasma nicotine - Self-administration

Mecamylamine is a centrally active nicotinic cholinergic antagonist with both a competitive and a noncompetitive component (Taylor 1980). It has been shown in laboratory experiments to block in a dose-related fashion a variety of subjective, behavioral, and physiological responses to nicotine in both humans and animals. Researchers from Jarvik in 1973 to Henningfield in 1984 have in fact argued for the potential usefulness of mecamylamine as a therapeutic agent, and a preliminary clinical trial conducted by Tennant et al. (1984) found it to be of some help.

Acute administration of mecamylamine, however, has also been shown to increase smoking behavior as reflected by topogaphical parameters - e.g., number of cigarettes smoked and total number of puffs - and by changes in carbon monoxide levels (Domino 1973; Nemeth-Coslett et al. 1986; Stolerman et al. 1973). This phenomenon suggests that smokers may engage in compensatory behavior in an attempt to counteract the effects of mecamylamine through self-titration. Alternatively, smokers may smoke more in order to achieve nicotine-produced relief from dysphoric effects of mecamylamine. In the present study, the effects of pretreatment with mecamylamine versus placebo upon levels of plasma nicotine were examined in the hopes of shedding light on these issues.

Offprint requests to: C.S. Pomerleau

\section{Materials and methods}

Subjects were 8 male smokers, healthy and not on medication, recruited from the community. To be included, they had to smoke at least 20 cigarettes per day and to have smoked for at least 5 years. Mean age was $45 \pm 5$ years. They had smoked for a mean of $30 \pm 5$ years and smoked a mean of $28 \pm 3$ cigarettes per day. Mean Fagerstrom Tolerance Questionnaire score was $7 \pm 1$ and mean plasma cotinine level $263.6 \pm 22.6 \mathrm{ng} / \mathrm{ml}$, characterizing them as moderately heavy smokers (Pomerleau et al. 1983).

On 2 days, separated by 1-3 days, subjects reported to the laboratory at 10:30 a.m. Blood pressure was measured upon arrival and again just before the session as an index of mecamylamine activity. Four subjects received $12.5 \mathrm{mg}$ mecamylamine PO the 1st day and placebo on the 2nd; the rest received placebo first and mecamylamine second. (This dose produced the most favorable effects-to-side-effects ratio in the pioneering study of Stolerman et al. in 1973.) An hour later, subjects were asked to smoke one usual-brand cigarette to ensure a comparable state of minimal deprivation. Just before the session, baseline subjective measures (Profile of Mood States, Spielberger State Anxiety Inventory, and Shiffman Withdrawal Questionnaire) were collected. A butterfly infusion set connected to a 1-m line was inserted into a left forearm vein; the line was heparinized and run through a channel in the wall for unobtrusive withdrawal of blood.

Sessions began 90 min after administration of drug or placebo. After a 5-min acclimation period, subjects smoked a high-nicotine research cigarette containing $2.9 \mathrm{mg}$ nicotine. A cigarette holder equipped with a pressure transducer allowed computerized collection of total puff volume and puff number. After $5 \mathrm{~min}$ had elapsed, they smoked another high-nicotine cigarette, following which they filled out subjective forms. Subjects were then asked to dip right hand and forearm into a tank of circulating water maintained at $3^{\circ} \mathrm{C}$, depressing a cradle that activated a timer. They were requested to nod when they became aware of the pain and to remove the arm from the tank when they could no longer stand the pain. This procedure served as a marker of nicotine activity, since nicotine produces reliable threshold increases for both awareness and tolerance of pain (Pomerleau et al. 1984). Blood samples were withdrawn at $0,5,10,15,20,25,30,35,45$, and $55 \mathrm{~min}$.

Data were subjected to repeated-measures ANOVA, using drug condition and time as repeated measures. 


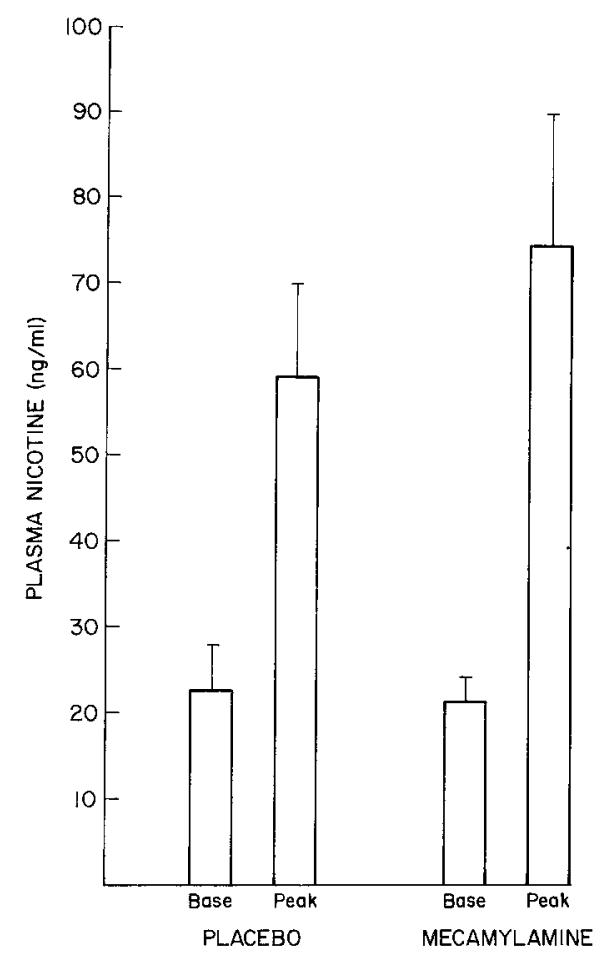

Fig. 1. Mean ( \pm SEM) base-to-peak plasma nicotine levels after two high-nicotine cigarettes following pretreatment with placebo versus pretreatment with mecamylamine; $N=8$

\section{Results}

Mecamylamine had a marked effect on systolic blood pressure: Although systolic blood pressure dropped between arrival and start of session on both the mecamylamine and placebo day, the decrease for mecamylamine was significantly greater than that for placebo $[F(1,7)=5.23, P<0.05$, 1-tailed]. Diastolic blood pressure drops were in the expected direction though not significant.

Baseline scores for the Spielberger and four of the six POMS subscales showed a tendency, though nonsignificant, towards greater dysphoria on the mecamylamine day. Three subscales of the Shiffman were scored separately (see Pomerleau et al. 1983); the (generalized) Discomfort subscale (querying calmness, concentration, contentedness, tenseness, and irritability) revealed significantly greater dysphoria on the mecamylamine day $[t(7)=2.411, P<0.05]$.

As shown in Fig. 1, base-to-peak elevations of plasma nicotine (using $\min 0$ as baseline and peak values determined individually for each subject on each day) were significantly greater on the mecamylamine day than on the placebo day $[F(1,7)=4.17, P<0.05,1$-tailed]. Two subjects achieved unusually high peak levels of nicotine $(111.0 \mathrm{ng} / \mathrm{ml}$ and $165.0 \mathrm{ng} / \mathrm{ml}$ ) following mecamylamine pretreatment. Subjects varied with respect to how much the mecamylamine treatment influenced subsequent nicotine intake. Figure 2 presents data for individual subjects in terms of their base-to-peak difference scores on the placebo day and the mecamylamine day.

The observed changes in plasma nicotine were not accompanied by significant differences in either total puff volume or total puff number. No systematic subjective changes between placebo and mecamylamine conditions were detected. Likewise, no significant differences were uncovered

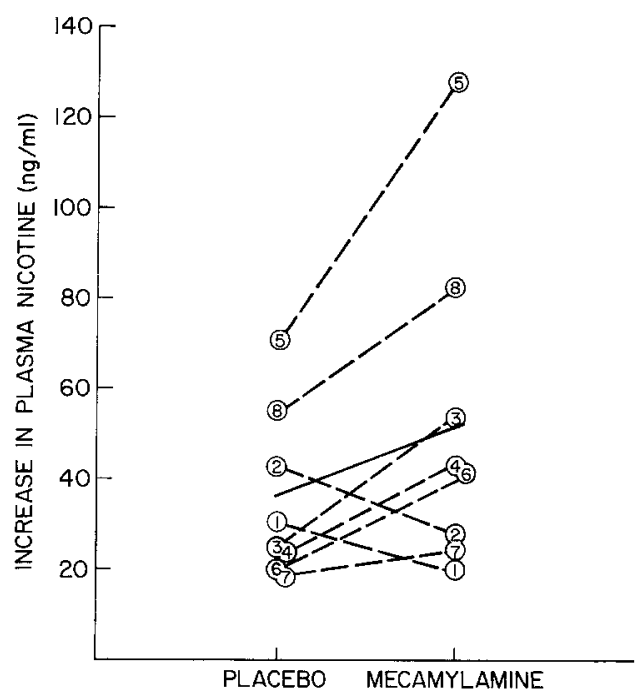

Fig. 2. Individual and mean base-to-peak increases in plasma nicotine levels following two high-nicotine cigarettes after pretreatment with placebo or mecamylamine; subjects identified by number

for pain-awareness threshold, endurance threshold, and scores on the McGill pain rating scale. No order effects were found for any measure.

\section{Discussion}

The obvious interpretation of the striking increases in plasma nicotine following mecamylamine pretreatment is that subjects were attempting to overcome the blockade, either to achieve relief from withdrawal or some other reinforcing effect of nicotine. An alternative explanation for the increase in smoking, as noted above, is that it represented an attempt to obtain nicotine-produced relief of mecamylamine-induced dysphoria. Baseline subjective scores did in fact tend to suggest greater dysphoria on the mecamylamine day than on the placebo day. It seems doubtful, however, that the extremely high plasma nicotine levels observed could be accounted for by such an explanation. This conclusion is supported by the finding of Stolerman et al. (1973) that pentolinium, a peripheral nicotinic cholinergic blocker used as a control, produced dysphoria equivalent to that of mecamylamine without concomitant changes in smoking behavior.

Whether or not subjects succeeded in overcoming the mecamylamine blockade cannot be definitively determined; more subtle indices of nicotine's psychological effects may be needed to settle the question. If complete or substantial blocking had been achieved, one would expect an initial increase in nicotine self-administration to be followed by a decrease. This has in fact been shown in monkeys (Goldberg et al. 1983). A similar demonstration over successive smoking trials in humans, using differing doses of mecamylamine and concomitant measures of plasma nicotine, would provide additional evidence regarding the clinical and ex- 
perimental usefulness of mecamylamine, especially in a selfdosing paradigm.

Following mecamylamine pretreatment, four of the eight subjects reached peak plasma nicotine levels in excess of $60 \mathrm{ng} / \mathrm{ml}$, the mean level at which induction of nausea was previously observed in our laboratory (Seyler et al. 1986), yet none reported nausea nor showed any objective signs of illness. Interestingly, Spealman and Goldberg, in a 1982 study of schedule-controlled behavior by intravenous injections of nicotine in squirrel monkeys, reported a similar finding; doses on the order of $300 \mu \mathrm{g} / \mathrm{kg}$ usually produced vomiting in these monkeys even at very low levels of responding, whereas in combination with mecamylamine, vomiting was not seen. These findings suggest that mecamylamine successfully blocked nicotininic cholinergic mechanisms involved in nicotine-induced nausea and vomiting and raise the question of whether exposure to potentially toxic levels of nicotine in the absence of normal selflimiting feedback mechanisms constitutes an added risk although hexamethonium, a quaternary ganglionic blocker, and pempidine, like mecamylamine a tertiary compound, seem to confer protection from nicotine toxicity in mice (Barrass et al. 1969).

Failure to obtain concomitant differences in smoking topography is surprising, since topographical changes have been related to nicotine intake (Herning et al. 1983), and since previous findings of increased smoking following mecamylamine are based on topographical data (e.g., NemethCoslett et al. 1986). Possibly with the use of high-nicotine cigarettes, even small differences in depth of inhalation might produce large differences in plasma nicotine levels. Indeed, it should be noted that cigarettes making large amounts of nicotine available to the smoker may be required to produce the exaggerated elevations observed in this study, since smoking of a usual-brand cigarette $1 \mathrm{~h}$ after mecamylamine administration failed to produce a difference in baseline nicotine measures collected $30 \mathrm{~min}$ later. Nevertheless, this outcome sounds a cautionary note with regard to relying entirely on topographical data to reach conclusions about nicotine intake, particularly in the presence of a pharmacological blocker.

In summary, the current findings confirm directly, for the first time, the inferences of previous investigators that smokers self-administer higher doses of nicotine after the administration of mecamylamine, at least on an acute basis. The extent to which this phenomenon compromises the effectiveness of mecamylamine as a blocker when given at safe doses, especially in a nicotine self-dosing paradigm where intake cannot be controlled, remains to be established.
Acknowledgements. The authors acknowledge with gratitude the assistance of Jean-Jacques Hajjar, Anthony Schork, and William Shanahan. Support for this research was provided by National Cancer Institute grant CA/DA 42730 to the second author.

\section{References}

Barrass BC, Blackburn JW, Brinblecombe RW, Rich P (1969) Modification of nicotine toxicity by pretreatment with different drugs. Biochem Pharmacol 18:2145-2152

Domino EF (1973) Neuropsychopharmacology of nicotine and tobacco smoking. In: Dunn WL, Jr. (ed) Smoking behavior: motives and incentives. Winston, Washington DC, pp 5-31

Goldberg SR, Spealman RD, Risner MD, Henningfield JE (1983) Control of behavior by intravenous nicotine injection in laboratory animals. Pharmacol Biochem Behav 19:1011-1020

Henningfield JE (1984) Pharmacologic basis and treatment of cigarette smoking. J Clin Psychiatry 45 [12, sec. 2]:24-34

Herning RI, Jones RT, Benowitz NL, Mines AH (1983) How a cigarette is smoked determines nicotine blood levels. Clin. Pharmacol Ther 33:84 90

Jarvik ME (1973) Further observations on nicotine as the reinforcing agent in smoking. In: Dunn WL (ed) Smoking behavior: motives and incentives. Winston, Washington DC, pp 33-49

Nemeth-Coslett R, Henningfield JE, O'Keeffe MK, Griffiths R R (1986) Effects of mecamylamine on human cigarette smoking and subjective ratings. Psychopharmacology 88:420 425

Pomerleau OF, Fertig JB, Shanahan SO (1983) Nicotine dependence in cigarette smoking: An empirically-based, multivariate model. Pharmacol Biochem Behav 19:291-299

Pomerleau OF, Turk DC, Fertig JB (1984) The effects of cigarette smoking on pain and anxiety. Addict Behav 9:265-271

Seyler LE Jr., Pomerleau OF, Fertig JB, Hunt D, Parker K (1986) Pituitary hormone response to cigarette smoking. Pharmacol Biochem Behav 24:159-162

Spealman RD, Goldberg RD (1982) Maintenance of schedule-controlled behavior by intravenous injections of nicotine in squirrel monkeys. J Pharmacol Exp Ther 223:402-408

Stolerman IP, Goldfarb T, Fink R, Jarvik ME (1973) Influencing cigarette smoking with nicotine antagonists. Psychopharmacologia 28:217-259

Taylor P (1980) Ganglionic stimulating and blocking agents. In: Gilman AG, Goodman LS, Gilman A (eds) The pharmacological basis of therapeutics (6th ed). MacMillan, New York, pp 211-219

Tennant FS, Tarver AL, Rawson RA (1984) Clinical evaluation of mecamylamine for withdrawal from nicotine dependence. In: Harris LS (ed) Problems of drug dependence. National Institute on Drug Abuse, Research Monograph, Volume 49, (ADM) 84-1316. Government Printing Office, Washington DC

Received October 28, 1986/Final version October 31, 1986 